UNIVERSIDADE FEDERAL DO ESTADO DO RIO DE JANEIRO - UNIRIO
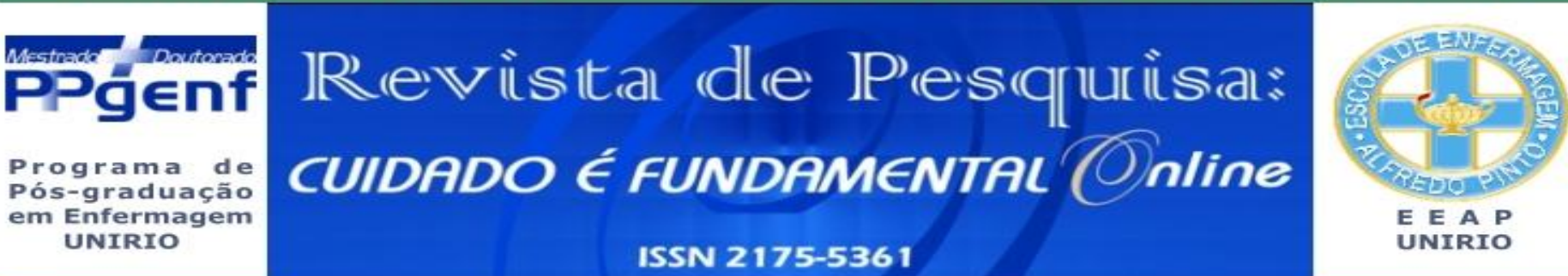

Ministério da Educação

\title{
PESQUISA
}

\section{PALLIATIVE CARE IN THE UTI: NURSES' UNDERSTANDING}

\author{
CUIDADOS PALIATIVOS NA UTI: COMPREENSÃO DOS ENFERMEIROS
}

\section{LOS CUIDADOS PALIATIVOS EM LA UTI:COMPRENSIÓN DE LOS ENFERMEROS}

Nara Calazans Balbino Barros ${ }^{1}$, Estela Rodrigues Paiva Alves ${ }^{2}$, Cecília Danielle Bezerra Oliveira ${ }^{3}$, Maria Djair Dias ${ }^{4}$, Inácia Sátiro Xavier de França ${ }^{5}$, Maria Eliane Moreira Freire ${ }^{6}$

\begin{abstract}
Objective: Investigate nurses' understanding of palliative care. Method: Qualitative study conducted in April and May 2011 with six nurses from the UTI of a public hospital in João Pessoa/PB. We used a questionnaire containing data characterizing the sample and three guiding questions. The data were analyzed according to the Collective Subject Discourse technique. The study was approved by the Ethics Committee of the College of Santa Emilia Rodat, $\mathrm{N}^{\circ}$ 014/2011. Result: Central ideas identified: 1) multidisciplinary actions to promote well-being of patients and their families by relieving pain and physical problems, psychosocial and spiritual. 2) actions that provide comfort and pain relief. 3) significant care and patient and famílianecessários year, by Profissionais of saúde, aiming morteumador and sofrimento week. Conclusion: With responses from nurses who flee to the essence of palliative care. Descriptors: Nursing, Intensive care units, Hospicecare.
\end{abstract}

\section{RESUMO}

Objetivo: Investigar a compreensão dos enfermeiros sobre cuidados paliativos. Método: Estudo qualitativo realizado emabrile maio de $2011 \mathrm{com}$ seis enfermeiras da UTI de um hospital público de João Pessoa/PB. Utilizou-se um questionário contendo dados de caracterização da amostra e três questões norteadoras. As informações foram analisadas conforme a técnica Discurso do Sujeito Coletivo.O estudofoi aprovado pelo Comitê de Ética em Pesquisa da Faculdade Santa Emília de Rodat, $n^{\circ}$ 014/2011. Resultados: Ideias centraisidentificadas:1) ações multiprofissionais que visam promover bem estar aos pacientes e seus familiares através do alívio da dor e de problemasfísicos, psicossociais e espirituais.2) ações que proporcionam conforto e alívio da dor. 3)cuidados importantes e necessários ao paciente e família, realizado por profissionais de saúde, visando uma morte sem dor e sofrimento. Conclusão: Parte dos enfermeiros apresentam respostas que fogem à essência do cuidado paliativo. Descritores: Enfermagem, Unidade de terapia intensiva, Cuidados paliativos.

\section{RESUMEN}

Objetivo: Investigar lacomprensión de lasenfermerassobre cuidados paliativos. Método: Estudiocualitativo realizado en abril y mayo de 2011 con seis enfermeras de laUTI de un hospital público en João Pessoa/PB. Se utilizóuncuestionarioconlacaracterización de lamuestra y tres preguntas orientadoras.Los datosfueronanalizadosutilizando la técnicadel Discurso delSujetoColectivo. Estudioaprobado por el Comité de Ética delColegio Santa EmiliaRodat, $\mathrm{n}^{\circ}$ 014/2011. Resultado: Ideascentrales:1) accionesmultidisciplinarias para promover elbienestar de los pacientes y sus familias por medio de los problemas de dolor de socorro y físicos, psicosociales y espirituales. 2) lasacciones que proporcionancomodidad y alivio deldolor. 3)cuidar a los pacientes y lasfamilias de losprofesionales de lasalud, en busca de una muertesindolor y elsufrimiento. Conclusión: Lasrespuestas de losenfermeroshuir a laesenciadel cuidado paliativo. Descriptores: Enfermería, Unidades de cuidados intensivos, Cuidados paliativos.

\footnotetext{
Especialista em Enfermagem em UTI pela Faculdade de Enfermagem Santa Emília de Rodat (FASER), Brasil. E-mail: naracalazans@hotmail.com. ${ }^{2}$ Mestre em Enfermagem em Promoção à Saúde pelo Programa Associado de Pós-Graduação da Universidade de Pernambuco e Universidade Estadual da Paraíba (PAPGEn-UPE/UEPB). Docente do Instituto de Educação Superior da Paraíba (IESP), Brasil. E-mail: rodrigues.estela@gmail.com. ${ }^{3}$ Enfermeira. Mestranda do Programa Associado de Pós-Graduação da Universidade de Pernambuco e Universidade Estadual da Paraíba (PAPGEn-UPE/UEPB), Brasil. E-mail: cecilia.dani@hotmail.com. ${ }^{4}$ Docente Associada do Departamento de Enfermagem Saúde Pública e do Programa de Pós-Graduação em Enfermagem da Universidade Federal da Paraíba (UFPB), Brasil. E-mail: mariadjair@yahoo.com.br. ${ }^{5}$ Docente do Departamento de Enfermagem da Universidade Estadual da Paraíba (UEPB) e Programa Associado de Pós-Graduação da Universidade de Pernambuco e Universidade Estadual da Paraíba (PAPGEn-UPE/UEPB), Brasil. E-mail: inacia_satiro@hotmail.com. ${ }^{6}$ Doutoranda do programa DINTER da Escola de Enfermagem de Ribeirão Preto (EERP/USP). Docente da Universidade Federal da Paraíba (DENC/UFPB), Brasil. E-mail: enf_elimoreira@hotmail.com.
} 


\section{INTRODUÇÃO}

O verbo paliar, do latim palliare, palium, significa em seu modo mais abrangente, proteger, cobrir com capa. No entanto, paliar é mais usado em nosso meio, como aliviar provisoriamente, remediar, revestir de falsa aparência, dissimular, bem como adiar, protelar. O cuidado paliativo (CP) ou paliativismo, é mais que um método, é uma filosofia do cuidar. O CP visa prevenir e aliviar o sofrimento humano em muitas de suas dimensões. ${ }^{1}$

Os cuidados paliativos são cuidados direcionados aos pacientes onde não existe a finalidade de curar, uma vez que a doença já se encontra em um estágio progressivo, irreversível e não responsivo ao tratamento curativo, sendo o objetivo desses cuidados propiciarem qualidade de vida nos momentos finais. ${ }^{2}$

A Organização Mundial da Saúde (OMS) defini cuidados paliativos como uma abordagem que melhora a qualidade de vida dos pacientes e de seus familiares, frente a doença terminal, através da prevenção e do alívio do sofrimento por meio da identificação precoce, avaliação religiosa e tratamento da dor e de outros problemas, físicos, psicossociais e espirituais. ${ }^{3}$

Para aprofundar a compreensão acerca de cuidados paliativos, o profissional de saúde precisa olhar para o cuidado mais de perto para contemplar as quatro necessidades humanas básicas: a de existir, a de pensar, a de sentir e a de agir no mundo. 0 cuidar é a pedra fundamental do respeito e do valor da dignidade humana. É no cuidar que mais se expressa a solidariedade para com os outros e é por isso quenesse estágio crítico de final de vida, toda a relação de terapêutica deveria estar fundamentada nesta ação. ${ }^{4}$
Acredita-se que a educação recebida pelos acadêmicos de enfermagem não permita acreditar que a morte possa acontecer na vigência de simples cuidados paliativos e que muitas vezes estes não estão preparados para esse tipo de assistência. ${ }^{5}$ A maioria dos enfermeiros já na sua atuação profissional acredita que nesta fase a função dos médicos é promover procedimentos e terapêuticas que mantenham o paciente "vivo" a qualquer custo.

A busca da qualidade de vida do paciente tem sido reiterada como uma das pedras angulares dos cuidados paliativos, havendo um número significativo e crescente de pesquisas sobre o que seria qualidade de vida em cuidados no fim da vida, e sobre quais seriam seus possíveis indicadores, especialmente para o paciente e seu cuidador. Esta é uma questão crucial, pois estudos mostram o quanto a preferência dos pacientes com doenças terminais em relação ao tratamento é pouco considerado pelos médicos, e o quanto há problemas de comunicação destes com seus pacientes, bem como o quanto há pouca atenção e pouco cuidado oferecido pelos médicos a seus pacientes e familiares neste período. ${ }^{6}$

O impacto da morte na sociedade e, em particular, entre os profissionais de saúde é frequentemente subestimado e pouco estudado. De maneira geral, os estudos que abordam esse tema são escassos. Além disso, não há um treinamento adequado dos enfermeiros para lidar com pacientes terminais e a morte. ${ }^{7}$

Tendo em vista a importância do tema, estudar a compreensão dos profissionais de enfermagem em uma UTI, sobre os cuidados paliativos é relevante. Pois mesmo com toda tecnologia disponível e aplicada no sentido de curar, ou prolongar a vida, pode-se encontrar uma diversidade de pacientes que não se beneficiam 
Barros NCB, Alves ERP, Oliveira CDB, et al. com essa medicina, assim só restando-lhe um cuidado mais específico.

Levando em consideração a importância do profissional de enfermagem nesses cuidados, abrimos uma discussão para responder aos seguintes questionamentos: qual a compreensão dos enfermeiros que atuam na UTI sobre cuidados paliativos? Que ações eles desenvolvem mediante pacientes terminais no contexto de cuidados paliativos? Qual a opinião dos enfermeiros da UTI acerca dos cuidados paliativos?

Face ao exposto, o presente estudo teve por objetivo verificar a compreensão de enfermeiros que atuam na UTI sobre os cuidados paliativos.

\section{METODOLOGIA}

Estudo exploratório-descritivo com abordagem qualitativa, realizado em uma UTI de um hospital público de João Pessoa/PB, envolvendo seis enfermeiros integrantes do quadro de pessoal do setor da instituição, que aceitaram participar livremente da pesquisa. A escolha do local se deu pela grande concentração da população alvo, viabilidade da investigação e por fim a acessibilidade.

Como critério de inclusão foi definido que os enfermeiros tivessem mais de um ano de experiência e atuação na UTI e disponibilidade para participar da pesquisa.

Para a coleta das informações foi utilizado um questionário semiestruturado, contendo questões de caracterização da amostra(idade, sexo, estado civil e tempo de trabalho na área) e trêsquestões norteadoras elaboradas pelos pesquisadores:1) Qual a sua compreensão sobre cuidados paliativos?;2)Que ações você executa e as considera como próprias dos cuidados
Palliative care in the UTI...

paliativos? e3) Qual sua opinião acerca destes cuidados no contexto de UTI?

Os dados foram coletados após todos os participantes assinarem o Termo de Consentimento Livre e Esclarecido, antecedidos de esclarecimentos sobre os propósitos da pesquisa, riscos e benefícios, processo de coleta dos dados e apresentação da carta de autorização para o desenvolvimento da pesquisa à coordenadora e aos enfermeiros da UTI.

A entrega dos questionários ocorreu respeitando a jornada de trabalho de cada enfermeiro, bem como o seu recolhimento, os quais foram respondidos por escrito e sem a presença da autora resguardando a privacidade e anonimato dos atores.

Tratando-se de um estudo qualitativo, a amostra foi alcançada conforme a saturação dos dados, portanto, não sendo uma condição, a delimitação do número de participantes.

A coleta foi realizada durante os meses de abril e maio de 2011. Os dados foram analisados com base no referencial teórico de Lefévre dispostos de acordo com a técnica do Discurso do Sujeito Coletivo (DSC). ${ }^{8}$ Tal análise é constituída por expressões chaves que tem a mesma ideia central ou ancoragem, ou seja, a soma de discursos/pensamentos. Para operacionalização desta técnica faz-se necessário os seguintes passos: seleção das expressões-chaves; identificação da ideia central de cada uma das expressões-chaves; identificação das ideias centrais semelhantes ou complementares, transcrevendo-se na íntegra os termos utilizados pelo participante do estudo e a reunião das expressões chave referente às ideias centrais em um discurso síntese, constituindo assim, o DSC. ${ }^{8}$

0 presente estudo considerou todos os aspectos éticos em pesquisas que envolvem seres humanos, no que diz respeito à ética, à privacidade, ao anonimato e ao direito de desistir 
Barros NCB, Alves ERP, Oliveira CDB, et al. da pesquisa em qualquer momento sem prejuízo para os participantes do estudo, normatizados pela Resolução 196/96 do Conselho Nacional de Saúde. ${ }^{9}$

\section{RESULTADOS E DISCUSSÃO DOS DADOS}

\section{CARACTERIZAÇÃO DA AMOSTRA}

A amostra contou com a participação de seis enfermeiros da UTI. De acordo com o instrumento de coleta de dados, destaca-se que, com relação à idade, três participantes tem idade entre 30 e 39 anos e três têm idade entre 40 a 49 anos. Em relação ao sexo dos participantes, cinco são do sexo feminino e um do sexo masculino. Quanto ao estado civil, cinco responderam serem solteiros e um afirmou ser casado.

Quanto ao tempo de trabalho na área, um respondeu que atua como enfermeiro há vinte e oito (28) anos; um afirmou que atua nessa área há dezenove (19) anos; um respondeu que trabalha há treze (13) anos na área; um relata que trabalha há sete (7) anos e outros dois responderam que atuam como enfermeiros há cinco (5) anos.

Com relação à carga horária semanal, um respondeu que trabalha setenta e duas horas semanais, dois disseram trabalhar trinta horas semanais, dois responderam que trabalham vinte e quatro horas semanais e um preferiu não responder. Em relação ao grau de instrução, três responderam que têm apenas a graduação em enfermagem e outros três responderam que têm especialização em UTI.

\section{DADOS RELATIVOS AOS OBJETIVOS PROPOSTOS}

Após leitura das respostas contidas no formulário utilizado para a coleta de dados, foi possivel identificar ideias centrais e expressões chaves das discussões dos sujeitos participantes,
Palliative care in the UTI...

recomendadas pela técnica de análise escolhida, possibilitando com isto, a elaboração do discurso do sujeito coletivo, apresentados a seguir, com suas respectivas discussões. ${ }^{8}$

Uma grande parte dos participantes desse estudo mostrou ideias contrárias, demonstrando em primeiro momento ideias confusas em relação ao conceito, algumas até fugindo totalmente da filosofia dos cuidados paliativos. Em outro momento percebem-se as dificuldades que eles tiveram em citar quais as ações paliativas mais comuns ou mais utilizadas em pacientes terminais, chegando a citar os cuidados básicos que enfermagem deve ter com os pacientes, mas que não são os cuidados específicos da medicina paliativa. Por fim, quando thes foram perguntado sobre a opinião deles acerca destes cuidados dentro do contexto de uma UTI, a maioria (cinco participantes) entrou em consenso ao falar da importância da aplicabilidade desses cuidados aos pacientes da UTI, respondendo assim de forma clara e coerente, mas entrando em contrariedade com algumas respostas ditas anteriormente.

\begin{tabular}{|c|c|}
\hline Ideia Central & Discurso do Sujeito Coletivo \\
\hline $\begin{array}{l}\text { Ações multiprofissionais que visam } \\
\text { promover bem estar aos pacientes e seus } \\
\text { familiares através do alívio da dor e de } \\
\text { problemas, físicos, psicossociais e } \\
\text { espirituais. }\end{array}$ & $\begin{array}{l}\text { [...]é a prática do atendimento, } \\
\text { envolvendo os aspectos físicos, } \\
\text { emocionais, sociais[...]; É um conjunto } \\
\text { de ações multiprofissionais que visa } \\
\text { oferecer conforto ao paciente e seus } \\
\text { familiares[...]; São cuidados ao bem } \\
\text { estar do paciente, e que nós como } \\
\text { enfermeiros fazemos sem autorização } \\
\text { médica[...]; Dara assistência precisa ao } \\
\text { cuidado em que há doença crônica e } \\
\text { dor[...]. }\end{array}$ \\
\hline Ações que não resultam em melhora. & $\begin{array}{l}{[\ldots] \text { são os primeiros atendimentos }} \\
\text { admissionais; São aqueles cuidados } \\
\text { prestados ao paciente enfermo, mas que } \\
\text { não resultará em melhora }[\ldots] .\end{array}$ \\
\hline
\end{tabular}

Figura 1- Ideia Central, DSC dos Enfermeiros em resposta a questão: Qual a sua compreensão sobre cuidados paliativos?

Fonte: Dados da pesquisa, 2011.

Dos discursos dos participantes mediante a questão "qual a sua compreensão sobre cuidados paliativos?", emergiram duas ideias centrais:1) Ações multiprofissionais que visam promover bem estar aos pacientes e seus familiares através do 
Barros NCB, Alves ERP, Oliveira CDB, et al. alívio da dor e de problemas, físicos, psicossociais e espirituais e 2) Ações que não resultam em melhora, dispostas na figura 1.

$\mathrm{Na}$ essência do conceito de cuidados paliativos, destaca-se o alívio dos sintomas, a dor e o sofrimento em doentes que sofrem de doenças crônico-degenerativas, ou estão em fase final. Esses cuidados tratam os pacientes como um todo e procura melhorar a sua qualidade de vida. Esses cuidados destinam-se a garantir cuidados aos doentes em condições que viabilizam e incentivam o paciente a viver uma vida de forma útil, produtiva e gratificante até o momento da sua morte. Tem como importância oferecer a reabilitação, em termos de saúde física, mental e espiritual e que não pode ser negligenciada. ${ }^{4}$

A forma mais simplificada para definir cuidados paliativos, são ações ativas e integrais prestadas a pacientes com doença progressiva e irreversível, e a seus familiares, sendo o foco do cuidado voltado para o controle da dor e demais sintomas de ordem física, psicológica, social e espiritual. ${ }^{10} \mathrm{~A}$ ideia de prestação de cuidados paliativos com qualidade é necessário e para tanto, o enfermeiro deve reconhecer quando uma pessoa se encontra em fase terminal e, juntamente com a família do paciente, estabeleça precocemente contato, garantindo a continuidade e, não se restringindo aos aspectos físicos dos cuidados. $^{7}$

Neste sentido, diante das literaturas aqui mencionadas podemos constatar que uma parte dos enfermeiros apresentou respostas coerentes ao que foi perguntado e outra parte não tem ideia do que é realmente o cuidado paliativo.

Assim, diante das descrições feitas pelos participantes da pesquisa, é possível verificar que a maioria dos entrevistados apresenta uma compreensão fragilizada no que diz respeito aos cuidados paliativos, uma vez que embora em uma das citações o entrevistado tenha conceituado de
Palliative care in the UTI...

forma correta o que seria os cuidados paliativos, em seguida exemplificou a ação de cuidados paliativos com "compressas frias para hipertermia".

Outro participante do estudo apenas relatou que é "Dar a assistência precisa ao cuidado em que há doença crônica e dor definitivamente acentuada". Nesse caso, a definição não está errada, mas está incompleta, pois estes cuidados abrangem muito mais do que foi dito, são cuidados praticados em pacientes com doença crônica ou em fase final de vida e eles não têm que apresentarem obrigatoriamente dor acentuada para que esses cuidados the sejam prestados.

Duas respostas fugiram totalmente do contexto dos cuidados paliativos, que foram os seguintes discursos: "São os primeiros atendimentos admissionais!”. Este discurso não está coerente, considerando que esse tipo de cuidado não se refere ao atendimento de admissão do paciente na instituição. A forma mais coerente seria dizer que este é o cuidado prestado ao paciente e a família do mesmo, desde sua admissão até o acompanhamento à fase de luto.

No tocante ao discurso "São aqueles cuidados prestados ao paciente enfermo, mas que não resultará em melhora" observa-se que na verdade os cuidados paliativos não oferecem cura, mas isso não implica dizer que não the ofereça melhora do estado do paciente, uma vez que poderá proporcionar qualidade de vida em sua finitude, com mais conforto e dignidade.

\begin{tabular}{|c|c|}
\hline Ideia Central & Discurso do Sujeito Coletivo \\
\hline $\begin{array}{l}\text { Ações que proporcionam conforto e o } \\
\text { alívio da dor. }\end{array}$ & 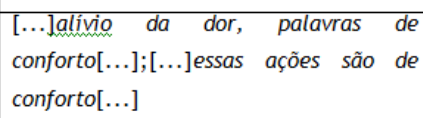 \\
\hline Ações de cuidado generalizado. & $\begin{array}{l}\text { [...]interação com a familia quanto na } \\
\text { documentação; [...]posicionamento do } \\
\text { paciente, compressas frias; [...]dor } \\
\text { precordial com antipânicos. Pós- } \\
\text { operatório de cirurgias; As ações } \\
\text { executadas em pacientes por exemplo } \\
\text { em ME[...]. }\end{array}$ \\
\hline
\end{tabular}


Barros NCB, Alves ERP, Oliveira CDB, et al.

Figura 2 - Ideia Central, DSC dos Enfermeiros em resposta a questão: Que ações você executa e as considera como próprias dos cuidados paliativos?

Fonte: Dados da pesquisa, 2011.

Aos participantes do estudo, foi perguntado se eles realizavam cuidados paliativos na UTI e cinco enfermeiros responderam que "sim", enquanto que apenas um respondeu que "não". Aos que responderam sim, perguntou-se: "que ações executavam e se achavam próprias dos cuidados paliativos?" Dos discursos obtidos dos participantes emergiram as ideias centrais: 1) Ações que proporcionam conforto e o alívio da dor e 2) Ações de cuidados generalizado.

Cada vez mais são identificadas metas que visam reduzir tensões éticas associadas ao tratamento de pacientes com doenças terminais, com ênfase ao tratamento baseado na obtenção do conforto. A promoção de uma melhor comunicação e do melhor conhecimento sobre cuidados paliativos nas UTI pode prevenir conflitos e melhorar o tratamento do paciente crítico. Sob este aspecto, é aconselhado que sejamrespeitados os aspectos socioculturais dos pacientes e de seus familiares e que também sejam avaliados os aspectos éticos e práticos da recusa ou suspensão de terapia fútil, da administração de sedoanalgésicos e da abordagem não farmacológica, que possam diminuir o sofrimento de todos os envolvidos no processo. ${ }^{10}$

Nesse ínterim, destacam-se as seguintes estratégias para o suporte da família: promoção de uma comunicação efetiva, envolvimento da família nos cuidados, negociação das necessidades especiais, providenciar um ótimo controle da dor e outros sintomas, providenciar suporte existencial, preparar a família para a morte, permitir que a família participe na morte e providenciar suporte para o luto. ${ }^{7}$

As ações paliativas como sendo necessidades individuais de cada paciente, ou seja, levando-se em consideração o estágio da R. pesq.: cuid. fundam. online 2013. jan./mar. 5(1):3293-01
Palliative care in the UTI...

doença (evolução, possíveis complicações, prognóstico a curto e médio prazo) ajustada às expectativas e valores da família/paciente, são instituídos visando atender às seguintes prioridades: identificar e excluir intervenções fúteis; prioridades terapêuticas; decisões antecipadas de final de vida e possíveis intercorrências; mudanças no ambiente; envolvimento da equipe multidisciplinar; enfrentando a doença em sua fase terminal; apoio à família na fase de luto. À medida que "o cuidar" passa a ser a prioridade é evidente que a analgesia e a sedação ganham uma atenção especial. ${ }^{11}$

Nos casos mais graves, a analgesia pode ser mantida com administração intermitente ou infusão contínua de opioides (morfina, fentanil) associada a analgésicos não opioides. Ao realizar alguma intervenção que promova dor (ex.: troca de drenos/sondas, mudança de decúbito, aspiração traqueal) uma dose suplementar de outro analgésico de curta duração pode ser adicionado. Algumas doenças em fase avançada (ex.: tumores com metástases ósseas), geram necessidades crescentes de analgesia. Do ponto de vista ético, moral e legal, não há como aceitar que o receio de efeitos colaterais impeça o uso de doses crescentes de opioides nessa situação. No sentido de obter sedação adequada pode ser utilizado sedativos leves, antipsicóticos, ou ainda a infusão contínua de dexmedetomidina. ${ }^{11}$

São várias as formas de executar esse tipo de ação paliativa, porém a mais importante é oferecer conforto ao paciente em seus momentos restantes de sua vida, no qual a maior queixa desses pacientes que se encontram em fase terminal é na sua maioria relativos à dor, e para o alívio desta, há uma diversidade de analgésicos e sedativos. A preferência ao tipo e quantidade de medicação a ser utilizada, irá depender do estado do paciente em relação ao grau de dor e o avanço da doença. 
Barros NCB, Alves ERP, Oliveira CDB, et al.

Outra ação que se destaca é em relação ao apoio que a equipe de saúde presta aos familiares e ao paciente, as informações acerca da doença fornecidas aos mesmos, por meio do diálogo é fundamental nesse tipo de serviço. Pois, tanto o paciente quanto os familiares passam a pensar em questões existências, pensam no processo vidamorte e com isso surge a inconformidade e muitas vezes a revolta. 0 apoio oferecido pela equipe deve estender-se até a fase de luto.

\begin{tabular}{|c|c|}
\hline Ideia Central & Discurso do Sujeito Coletivo \\
\hline $\begin{array}{l}\text { São cuidados importantes e necessários } \\
\text { ao paciente e familia, realizado por } \\
\text { profissionais de saúde, visando uma } \\
\text { morte sem dor e sofrimento. }\end{array}$ & $\begin{array}{l}\text { [...]lacho importante o envolvimento } \\
\text { multiprofissional junto ao paciente e a } \\
\text { família, visando uma morte sem dor e } \\
\text { tanto sofrimento[...]; Esse cuidado é } \\
\text { visto como complemento da } \\
\text { assistência[...]; E de vital importância, } \\
\text { pois a gravidade dos pacientes com dores } \\
\text { agudas e crônicas cabe ser observado } \\
\text { pelo profissional de saúde e tomar as } \\
\text { providências cabíveis[...]; São } \\
\text { necessários, pois a enfermagem está } \\
\text { intimamente ligada aos cuidados[...] }\end{array}$ \\
\hline
\end{tabular}

Figura 3 - Ideia Central, DSC dos Enfermeiros em resposta a questão: Qual sua opinião acerca destes cuidados no contexto de UTI?

Fonte: Dados da pesquisa, 2011.

Mais da metade dos participantes do estudo concordaram sobre a opinião acerca dos cuidados paliativos aplicados em pacientes internos na UTI, conforme as falas da figura 3.

As respostas supracitadas foram tão claras e concisas podendo ser consideradas mais precisas e melhores do que os conceitos referidos pelos mesmos anteriormente, na figura 1. Mostrando-se contrário a algumas respostas ditas anteriormente, revelando certa confusão em relação a sua compreensão acerca desses cuidados. 0 que nos faz perceber que os mesmos têm noção do que se trata, quando se fala em cuidados paliativos, mas que talvez não consigam expressar o seu conhecimento acerca deste tema.

Um dos participantes afirmou que: “Estes cuidados em UTI são pouco utilizados vez que estes pacientes que necessitam já chegam bastante graves praticamente em fase final, portanto são poucos que recebem esse tipo de
Palliative care in the UTI...

serviço em UTI”. Essa resposta não foi coerente com a filosofia dos cuidados paliativos, já que esses cuidados devem ser ofertados a todos os pacientes que se encontram na UTI, como já foi dito anteriormente e devem ser oferecidos principalmente a esse tipo de paciente, os que se encontram em fase terminal.

$\mathrm{Na}$ UTI, a integração entre os cuidados paliativos e curativos desde o momento da admissão vem sendo ressaltados cada vez com maior importância, na busca de um atendimento de qualidade. Vale ressaltar que em pacientes sob tratamento ambulatorial, o início precoce dos cuidados paliativos integrado aos cuidados modificadores de doença foi associado tanto à melhor qualidade de vida, quanto a importante ganho de sobrevida. ${ }^{10}$

Não resta dúvida sobre a importância da educação e do treinamento dos profissionais para a implantação de cuidados paliativos nas UTIs, cuidados esses que podem levar a diminuição do tempo de internação e a melhor qualidade do atendimento nesses setores. Diferentes modelos têm sido propostos para a melhoria dos cuidados paliativos nas UTI. Destacam-se dois modelos: o integrativo, que visa incorporar os cuidados e intervenções paliativas na prática diária dos intensivistas, e o consultivo, que ocorre por meio de interconsultas com equipes de cuidados paliativos, incorporado à prática dos cuidados em UTI. Além dos pacientes, tais cuidados devem ser fornecidos para os familiares do paciente. ${ }^{10}$

\section{CONSIDERAÇÕES FINAIS}

O estudo revelou que os cuidados paliativos são reconhecidos como uma importante questão de saúde pública, pois trabalha com o sofrimento, a dignidade, o cuidado das necessidades humanas e qualidade de vida das pessoas afetadas por uma 
Barros NCB, Alves ERP, Oliveira CDB, et al. doença crônica e degenerativa ou em fase final da sua vida. Com isso, a preocupação não se limita ao paciente que está sob os cuidados, mas também com seus familiares e amigos.

Observou-se a necessidade de uma formação (pós-graduação), na área de cuidados paliativos, pois o profissional de saúde que possui apenas uma formação básica (graduação), ainda não está preparado para enfrentar situações em que encontrem pacientes fora de possibilidades terapêuticas de cura, ou seja, pacientes em fase final de vida.

Assim, os cursos de formação de enfermeiros carecem de disciplinas que abordem os temas de morte especificamente, do luto e do morrer, que conduzam esse profissional para além do conhecimento técnico-científico adquirido. Para que o mesmo possa oferecer uma assistência humanizada, que priorize a dignidade humana.

No tocante à compreensão sobre cuidados paliativos, parte dos enfermeiros apresentou ideias coerentes sobre este cuidado, enquanto que outros apresentaram respostas que fogem à essência e filosofia deste modo terapêutico. Com relação às ações de cuidados paliativos que estes enfermeiros realizam, verificou-se que poucos atuam na perspectiva do alívio da dor e promoção do conforto.

Contudo, percebe-se claramente que esses profissionais necessitam rever novos conceitos sobre cuidados paliativos, para que se possa ter uma melhor compreensão acerca desses cuidados, como também devem ser realizados novos treinamentos com essa equipe de saúde.

Esse estudo é mais uma contribuição para os profissionais de saúde que atuam em UTI e um incentivo para que os mesmo procurem abordar ainda mais essa temática, visto que é de fundamental importância a presença de profissionais especializados nessa área. Desta forma, se faz necessário novos estudos,
Palliative care in the UTI...

intervenções e treinamentos para os profissionais, buscando assim, uma melhoria no sistema desses cuidados.

\section{REFERÊNCIAS}

1. Costa Filho RC, CostaJLF, GutierrezFLBR, MesquitaAF. Como Implantar Cuidados de Qualidade na Unidade de Terapia Intensiva. Rev. bras. ter.intensiva[periódico na internet] 2008 [acesso em 2012Abr 19];20(1):88-92. Disponível

em:http://www.scielo.br/pdf/rbti/v20n1/a14 v20n1.pdf.

2. Silva EP, SudigurskyD. Concepções sobre cuidados paliativos: revisão bibliográfica. Acta paul. enferm. [periódico na internet] 2008 [acesso em 2012 Abr 19];21(3):504-08. Disponível em: http://www.scielo.br/pdf/ape/v21n3/pt_20. pdf.

3. Floriani CA, SchrammFR. Desafios morais e operacionais da inclusão dos cuidados paliativos na rede de atenção básica. Cad. Saúde Pública [periódico na internet] 2007 [acessoem $2012 \quad \mathrm{Abr}$ 19];23(9):2072-80. Disponível em: http://www.scielo.br/pdf/csp/v23n9/08.pdf.

4. Pessini L, BertachiniL. Novas perspectivas em cuidados paliativos. Acta Bioethica.2006;12(2):231-42.

5. Bernier J, Hirdes A. O preparo dos acadêmicos de enfermagem brasileiros para vivenciarem o processo morte-morrer. Texto Contexto Enferm. [periódico na internet] 2007 [acesso em 2012 Abr 19];16(1):89-96. Dispon[ivel em: http://www.scielo.br/pdf/tce/v16n1/a11v16 $\mathrm{n} 1$.

6. Floriani CA. Cuidados no domicílio: desafios aos cuidados de crianças dependentes de 
Barros NCB, Alves ERP, Oliveira CDB, et al. tecnologia. J. Pediatr. [periódico na internet] 2010 [acesso em 2012 Abr 17];86(1):15-9. Disponível em: http: //www.scielo.br/pdf/jped/v86n1/v86n1 a04.pdf.

7. Fonseca JVC, Rebelo T. Necessidades de cuidados de enfermagem do cuidador da pessoa sob cuidados paliativos. RevBras. Enfer. [periódico na internet] 2011 [acesso em 2012 Abr 12];24(1):180-4. Disponível em: http: / /www.scielo.br/pdf/reben/v64n1/v64n 1a26.pdf.

8. Lefèvre $F$, Lefèvre $A M C$. $O$ sujeito coletivo que fala. Interface (Botucatu) [periódico na internet] 2006 [acesso em 2012 Abr 12];10(20):517-24. Disponível em: http: / /www.scielo.br/pdf/icse/v10n20/17.pd f.

9. Brasil. Conselho Nacional de Saúde. Diretrizes e normas regulamentadoras de pesquisa envolvendo seres humanos. Resolução 196 em 10 de outubro de 1996.

10. Moritz RD, Lago PM, Souza RP, Silva NB, Menses FA, Othero JCB et al. Terminalidade e cuidados paliativos na unidade de terapia intensiva. Rev. bras. ter. intensiva[periódico na internet] 2008 [acesso em 2012 Abr10];20(4):422-28. Disponível em: http://www.scielo.br/pdf/rbti/v20n4/v20n4a 16.pdf.

11. Piva JP, Garcia PCR, Lago PM. Dilemas e dificuldades envolvendo decisões de final de vida e oferta de cuidados paliativos em pediatria. Rev. bras. ter. intensiva[periódico na internet] 2011 [acesso em 2012 Abr10];23(1):78-86. Disponível em: http://www.scielo.br/pdf/rbti/v23n1/a13v23 n1.pdf.

12. Siqueira AL, Silva FM, Paula VG. The nursing and palliative care to patients through the spirituality of no treatment possibility.R.
Palliative care in the UTI... pesq.: cuid. fundam.[periódico na internet] 2011 [acesso em 2012 Abr 10];3(4). Disponível em:

http://www.seer.unirio.br/index.php/cuidad ofundamental/article/view/1501/pdf_447.

Recebido em: 19/04/2012

Aprovado em: 02/08/2012 